\title{
Review on antibiotics treatment by electrochemical membrane reactor
}

\author{
Lei Chao ${ }^{1}$, Feilong $\mathrm{Chen}^{1, *}$, Yi Han ${ }^{1}$ and Yafeng $\mathrm{Li}^{1}$ \\ ${ }^{1}$ Shenyang Jianzhu University, Municipal and Environmental Engineering Department, 110168 Shenyang Liaoning, China
}

\begin{abstract}
Lower consumption, higher efficiency, environmental protection, and reliability are the development trends for the treatment of antibiotic wastewater in future. To accomplish this, the electrochemical membrane reactor (ECMR) is developed by combining membrane filtration and electrochemical advanced oxidation technology. The device configuration and working mode of the electrochemical membrane reactor are introduced and compared. Besides, the principles of the removal of antibiotics by the reactor are explained with emphasis. Furthermore, the commonly used cathode and anode materials of the reactor in the current research are summarized, and the electrode materials are discussed. The effects of selection and modification on the elimination of antibiotics in the reactor and the impact are analysed. To address the limitations of electrochemical membrane reactors, this review proposes that more research should be done in the aspects of antibiotic degradation mechanism, reduction of membrane electrode R\&D costs, and actual application of amplification devices.
\end{abstract}

\section{Introduction}

Over the past several years, antibiotics have been attracted multiplying attention as a new type of hazardous bioactive pollutants. The main sources of antibiotics in water bodies are pharmaceutical wastewater, aquaculture, and incomplete metabolism of humans or livestock. Since the conventional wastewater treatment processes (activated sludge process, biofilm process, etc.) are mainly dependent on microbial life activities, emerging threats and challenges are forming in the following two aspects: 1) a significant inhibition of antibiotics on microorganisms makes it difficult to be oxidized and decomposed. Besides, antibiotics also reduce the effluent quality by destroying normal microflora; 2) potential microbial acclimation effect of antibiotic increasingly induce generation of bacterial resistance genes (ARGs). Once discharged into the aquatic environment, ARGs may produce serious biological hazard [1]. Therefore, research on preventing the continuous introduction of antibiotics and developing novel antibiotic removal technology, especially at a lower concentration, has become a prevalent hotspot for a while.

In recent years, the antibiotic removal technologies can mainly be divided into biological method, adsorption, membrane filtration, advanced oxidation technology [24], and the coupling and combination of various technologies. Although the cost of biological method and adsorption are relatively lower, the unstable removal efficiency highlights their inherent drawbacks. As for Membrane filtration, inevitable membrane fouling pollution and short lifetime seriously restrict its wide application on a large scale. Advanced oxidation technology, especially electrochemical advanced oxidation technology, benefits from numerous advantages, such as environmental-friendly operation, high-efficient removal rate, without additional reagents, and convenient facilities. However, electrode production cost and energy consumption limit its potential for development [5].

With these backgrounds, electrochemical membrane reactor is proposed via the combination of membrane filtration and the electrochemical advanced oxidation technology. In general, using a membrane electrode can entrap contaminants extensively and meanwhile reinforce mass transfer. Furthermore, electrochemical advanced oxidation process can reduce membrane fouling reversely. These characteristics reach the purpose of lower energy consumption and higher efficiency removal rate of contaminants [6].

This study focuses on the technique principles of antibiotic degradation in ECMR and emphatically introduces the technology and factors, such as current density, initial concentration, electrode pore size, $\mathrm{pH}$, residence time and electrolyte. Moreover, electrochemical membrane reactor configurations and conventional membrane electrode materials are summarized,

\section{Overview of ECMR}

Electrochemical membrane reactor, also known as filtertype electrochemical reactor, is a state-of-the-art device coupled membrane filtration technique with the electrochemical advanced oxidation reaction, which

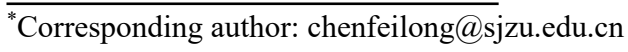


enhances mass transport effect and ability to eliminate antibiotics. The critical key point is a membrane electrode with both conductive and selective membrane filtration function. Membrane electrode is also known as filter electrode. Its conductive function is achieved by using electrode materials with good conductivity, such as carbon felt, carbon nanotubes, graphene, activated carbon fiber, various metal electrodes or metal oxide electrodes, while the filtering function is mainly achieved by selecting porous materials with specific porosity, such as graphite felt and foam metal [7]. The evaluation system of electrochemical membrane reactor was established from the device configuration, working mode and the main mechanism of degradation of antibiotics.

\subsection{Device configuration of ECMR}

In the conventional system, electrochemical reactor is a device that uses cathode and anode plates as conductive or catalytic medium to transfer electrons between contaminants and electrodes to achieve direct or indirect degradation and mineralization of pollutants. Since its cathode and anode plates are usually made of nonfiltering materials (such as graphite, stainless steel plate, titanium plate, platinum plate, etc.), the problems such as less electrochemical active sites, lower mass transfer efficiency, larger energy consumption and more side reactions have been criticized by the industry for a long time. Therefore, Wang et al. pointed out that researchers can explore more in the design of reasonable reactor configuration, the selection of excellent electrode materials and the attempt to modify the electrode in various aspects [4]. After persistent exploration and improvement, the appearance of the reactor components is diverse. While according to the shape of the reactor configuration, working electrode of ECMR can be divided into two types: flat plate and tubular.

The flat plate filter electrode is the most common form, which is generally a circular or rectangular with a specific thickness and porosity. Meanwhile, it has the characteristics of high mechanical strength and wide applicability. HUI et al. loaded nano-manganese oxides onto porous titanium to prepare $\mathrm{MnOx} / \mathrm{Ti}$ membrane electrode by sol-gel method and applied it to the electrocatalytic membrane reactor (ECMR) to achieve pollutant removal at low energy consumption $(0.25 \mathrm{kWh} / \mathrm{kg}$ COD) [8]. Similarly, Chen et al. firstly loaded the enhanced nanotube (ENTA) array on the titanium foam as the intermediate layer, and then loaded the tin-antimony metal oxide. Finally, the MP-Ti-ENTA/SnO2-Sb anode was prepared, which was used as the anode oxidation. The degradation rate of methyl isothiazolone can reach $96.7 \%$ within $120 \mathrm{~min}$ in the $\mathrm{NaCl}$ electrolyte system [9]. Therefore, flat-plate filter electrode is widely used. The membrane electrode with titanium foam as the substrate can achieve a good removal of antibiotics under the action of electrocatalytic oxidation after loading metal oxides or other modification and doping.

The tubular filter electrode is a hollow circular tube with a specific diameter and thickness. Generally, one end is closed to prevent short flow, and the other end is connected to the suction pump to provide continuous negative pressure. Yin et al [10] selected the low cost coal as the matrix conductive material, after crushing, removing oil pollution impurities and other pre-treatment, mechanical extrusion moulding was carried out, and finally produced tubular coal-based carbon film through the specific sintering process method, the average pore size of the carbon film was $0.382 \mathrm{um}$, the porosity was $49.56 \%$. Li et al $[8,11-13]$ finished more research on tubular titanium membrane in the past. Since the outer layer of titanium membrane anode is liable to be oxidized to semiconductor material $\mathrm{TiO} 2$, which reduce the conductivity, they have carried out many loading modification treatments on titanium foam, such as nano$\mathrm{MnOx}$, nano-V2O5, and $\mathrm{Co} 3 \mathrm{O} 4$ nanotubes. In addition, they have carried out catalytic conversion or degradation experiments on many contaminants, including oily wastewater, phenol, and alcohols, and have achieved great degradation rates. However, their recent studies have pointed out that the length and diameter of tubular membrane electrode may limit the fluid state of the solution in the reactor and affect the reaction efficiency. There are still some challenges such as mechanical strength deviation and less packing density, which need to confront.

\subsection{Working mode of ECMR}

The most prominent characteristic of ECMR is that it can operate at low current density, which depends on the working mode of filter electrode. Depending on whether the liquid flow in the reactor passes through the electrode, the reactor can be divided into two working modes: Flow-by and Flow-through [14].

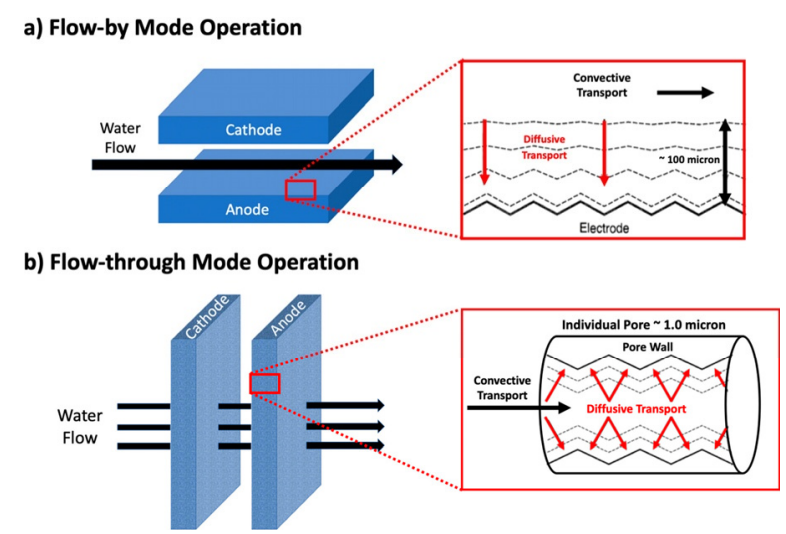

Figure.1 Schematic diagram of Flow-by(a) and Flowthrough(b) mode operation[15]

The traditional electrode working mode is Flow-by [Figure.1(a)], the liquid occurs mass transport and electron transfer on the electrode surface, so it can be limited by the concentration polarization in the diffusion boundary layer. While the internal penetration mode is the working mode of the filter electrode [Figure.1(b)]. The liquid flow passes through the electrode through the pore structure inside the electrode, which can reduce the influence of concentration polarization to a large extent. 
Chaplin pointed that the degradation of pollutants in the Flow-by mode was limited by diffusion at low current density $(<5 \mathrm{~mA} / \mathrm{cm} 2)$. Since the length scale of electrode roughness was much smaller than that of diffusion boundary layer, even high specific surface area electrode could only moderately improve the removal rate of pollutants. However, when the liquid flows through the pores of the electrode, and the thickness of the diffusion boundary layer can be compressed to a distance equivalent to the radius of the pore in the Flowthrough working mode[15]. Therefore, the selection of the filter electrode with high specific surface area can ensure that the surface area of most electrodes is electrically active at low current density, so the order of the reaction rate constant can be greatly improved.

\subsection{Degradation mechanism of ECMR}

The mechanism of antibiotic degradation in ECMR mentioned in the known reports mainly includes two steps: electro-adsorption and electrochemical oxidation. After the voltage is applied to the porous electrode, the electrode first adsorbs and aggregates antibiotics, and then eliminates pollutants directly or indirectly by electro-catalytic oxidation, which are also called direct oxidation and indirect oxidation. Direct oxidation refers to the phenomenon that the pollutants migrate to the two poles under the action of electric field and directly degrade into small molecular compounds by electron gain and loss on the surface of the electrode (anode), and finally are completely mineralized. Indirect oxidation refers to the non-selective degradation of organic pollutants by forming highly oxidizing active substances (such as hydroxyl radicals and superoxide radicals) to achieve the aim of contaminant mineralization [16]. In electrochemical reactors, direct oxidation and indirect oxidation usually coexist, but most research results show that indirect oxidation generally dominates. Moreover, according to the different electrolytes in the reaction system, the types of active substances produced by the free radicals which play a key role in indirect oxidation are also distinct, which directly affect the efficiency and removal mechanism of electrochemical reactor [17] According to the classification of the main contribution electrode, the working mechanism of electrochemical reactor can be divided into membrane cathode, membrane anode and duad-membrane electrode.

Membrane cathode refers to the functional electrode with both filtration and conductivity as the cathode, while the anode adopts non-filtration electrode, which only plays the role of forming a closed loop or anodic catalytic oxidation. The reported cathode matrix materials include graphite felt (GF), carbon felt (CF), carbon nanotubes (CNTs), coal, and so on. Carbon black, PTFE, and graphene are generally used to modify the cathode. Since semi-electron reduction reaction can occur at the cathode, the filter cathode is widely used in the nitrate removal. When in the degradation of antibiotics, it mainly relies on the cathode to produce active substances or intermediate products $(\cdot \mathrm{OH})$ with strong oxidizability. Therefore, the starting point of membrane cathode is to combine with other electrochemical advanced oxidation technologies such as electro-Fenton (EF), and enhance the yield of active substances by enhancing mass transfer of electrodes. Zhou et al prepared the CB-PTFE/GF electrode by loading polytetrafluoroethylene and carbon black onto graphite felt. The CB-PTFE/GF electrode was used as cathode to produce $\mathrm{H} 2 \mathrm{O} 2$ in situ. Since the internal pore structure of the filter electrode strengthened the dielectronic reaction of oxygen on the surface and inside of the electrode, the yield of $\mathrm{H} 2 \mathrm{O} 2$ was greatly increased under the condition of three-phase interface. Therefore, more hydroxyl radicals could be produced in the electroFenton system, and the removal rate of tetracycline reached $90 \%$, while the energy consumption was only $48.6 \mathrm{kWh} /$ (kg TOC) [18].

Membrane anode means that the anode is a membrane electrode with filtration function, which generally relies on anodic oxidation or catalytic oxidation to remove antibiotics. Compared with the membrane cathode, it has the advantages of not requiring additional reagents and diverse choices. The reported anode materials mainly include carbon nanotubes, metal foam (titanium foam, aluminium foam, Ti4O7), metal oxide electrode, BDD electrode, etc. Carbon nanotubesbased anodes are prone to electrochemical combustion and other phenomena to shorten the service lifetime. Similarly, metal anodes may occur electrochemical corrosion or passivation affect the performance of the electrode. Therefore, varied transition metals and their oxides are added to modify the anode to improve the oxidation of pollutants or the ability to produce hydroxyl radicals. In the study of tetracycline antibiotics removal by $\mathrm{Ti} 4 \mathrm{O} 7$ porous anode, Liang et al proposed that antibiotics removal mainly via two paths: direct oxidation at low anode potential; While when the anodic potential was greater than $2.38 \mathrm{~V}$ (vs SCE) (the standard potential of $\mathrm{OH}$ generated by water electrolysis), the indirect oxidation was mainly mediated by hydroxyl radicals. Especially in Magnéli phase Ti4O7 inactive anode', hydroxyl radicals were weakly adsorbed on the surface of the electrode through physical interaction, so that antibiotics were more effectively oxidized through free radical chain reaction, which distinguished from the hydroxyl radicals of 'active anode' [19] .

Duad-membrane electrode means that both cathode and anode are filter electrodes, which is rarely reported at present. The intention of duad-membrane electrode is to couple the membrane cathode and membrane anode into the same reactor to make full use of the excellent removal ability of the two electrodes for antibiotics. However, it was found that the double-membrane electrode reactor would have a large current fluctuation or even no current under closed conditions. The main reason is that the inevitable electrode side reaction produces gas and blocking affects the rapid response of current between cathode and anode. Therefore, the research on the double-film electrode is at the beginning stage and has great application potential. 


\section{Variable factors of ECMR}

\subsection{Current density}

Current density of the ECMR system is the most remarkable influence factors. Generally, rational current density may accelerate the moving speed of electrons and increase the electron transfer between the electrode and contaminants, enhancing the active substance generation amount, thereby improving degradation rate and mineralization rate of pollutants. Whereas after the current density exceeds a certain value, ultra-high current may lead to evolution of hydrogen, oxygen evolution and other various competing side reactions and decrease energy consumption efficiency eventually.

Wang et al pointed that the amount of hydroxyl radicals was closely relevant with the applied current density and played an important role in the electrochemical degradation of organic compounds. By setting a series of concentration gradient $(10,20,30$, and $40 \mathrm{~mA} / \mathrm{cm} 2$ ), they carried out a ciprofloxacin degradation experiment with an initial concentration of $50 \mathrm{mg} / \mathrm{L}$. With the increase of applied current density (I $\leqslant 30 \mathrm{~mA} / \mathrm{cm} 2$ ), the removal rate of ciprofloxacin was improved. However, when the applied current density continued to increase to $40 \mathrm{~mA} / \mathrm{cm} 2$, the removal and mineralization rate of ciprofloxacin decreased. The reason was that the higher applied current density not only increased the oxygen evolution reaction, but also caused the electrolyte SO42- to decompose into S2O82-. All in all, lower current density is more sensible for antibiotic removal [20].

\subsection{Initial concentration}

As for the electrochemical membrane reactor, the size of the filter electrode is determined, which means that the number of active sites of the electrode is fixed. Therefore, the loading of antibiotic concentration will affect the electrode activity. In the current research, it is generally used to treat low-concentration antibiotic wastewater. With the increase of antibiotic concentration load, the degradation efficiency of antibiotics generally decline, mainly due to three aspects: Firstly, under the condition of a certain current density, the number of active defects produced by the electrode is certain, and the amount of antibiotics can be treated at the same time is certain. Secondly, intermediate products or by-products can further inhibit the degradation of antibiotics by competing with the active sites of antibiotics. Thirdly, with the increase of concentration, the inactivation of electrode active sites may be irreversible [21]. Li et al. confirmed the three views by setting different concentrations $(100,200$ and $500 \mathrm{mg} / \mathrm{L})$ to study the electrochemical degradation of pyridine at a current density of $20 \mathrm{~mA} / \mathrm{cm} 2$. It was found that when the concentration was $100 \mathrm{mg} / \mathrm{L}$, the removal rate could reach $52 \%$. However, when the concentration increased to $500 \mathrm{mg} / \mathrm{L}$, the removal rate of pyridine eventually dropped to $25 \%$. It is believed that hydroxyl radicals generated by the electrocatalytic reaction is limited, and the degradation efficiency may decrease with the increase of the initial concentration of pyridine [22].

\subsection{Electrode pore size}

The pore size of the electrode mainly affects the specific surface area of the electrode. For the filter electrode, larger surface area means that the electrode can provide more active sites in the same volume. Chen et al studied the porous titanium films with different pore size distribution (10 um, $20 \mathrm{um}, 50 \mathrm{um}$ ), and found that the smaller the pore size, the larger the corresponding pore surface area, which was 48 433 times higher than that of the flat electrode with the same specification. Theoretically, the larger the specific surface area is, the larger the load of the electrode is. However, it should be noted that the general porous materials are usually used as the substrate of the electrode. In the process of loading the catalytic layer, the smaller the pore size is, the more likely the channel blockage occurs in the electrode matrix, thereby affecting the load per unit mass [9]. There are few studies on the removal rate of antibiotics with the electrode aperture as the influencing factor, and it is essential to explore the influence of the electrode aperture.

\section{4. $\mathrm{pH}$}

The initial pH of ECMR system should be set reasonably according to the degradation mechanism. XU et al set the initial $\mathrm{pH}$ to 3.0, 5.0, 9.0 and 11.0 to determine the effect of initial $\mathrm{pH}$ on naproxen degradation efficiency. It was found that when the initial $\mathrm{pH}$ increased from 3.0 to 11.0, the degradation rate of naproxen decreased from $96.6 \%$ to $50 \%$ in $4.56 \mathrm{~min}$. Naturally, they believed that the electrochemical degradation of naproxen at acidic $\mathrm{pH}$ was more favourable than that at alkaline $\mathrm{pH}$. On the one hand, $\cdot \mathrm{OH}$ is more likely to be generated at low $\mathrm{pH}$ $(\mathrm{pH}<9.0)$; On the other hand, the oxidation potential of $\cdot \mathrm{OH}$ is higher in acidic condition $(2.85 \mathrm{~V})$ than in alkaline condition $(2.02 \mathrm{~V})$, and higher $\mathrm{pH}$ may enhance the oxygen evolution reaction on the surface of porous $\mathrm{Ti} / \mathrm{SbO} 2-\mathrm{Sb} / \mathrm{Ce}-\mathrm{PbO} 2$, thus hindering the degradation of naproxen [23]. LIU et al studied the removal of tetracycline in EF system, which confirmed this phenomenon. They also demonstrated that the removal rate of tetracycline was $92.5 \%$ at $\mathrm{pH} 3.2$ and the removal rate decreased slightly at neutral $\mathrm{pH}(<8 \%)$. While at $\mathrm{pH} 10.6$, the removal rate decreased four times than that at $\mathrm{pH}$ 3.2. And in addition, the removal rate of TOC dropped from $37.4 \%$ to $2.3 \%$ [24].

\subsection{Residence time}

Generally, the setting of reactor flow rate determines the hydraulic retention time of pollutants. In theory, as for unidirectional flow reactor, the longer the retention time is, the more sufficient the contact between antibiotics and electrodes is, and the higher the removal rate is. But for the circulating reactor, shorter residence time can enhance stronger mass transfer and produce more active 
substances and the degradation of antibiotics is more favourable. Under the fixed working volume of the reactor, REN et al achieved the degradation efficiency of about $100 \%$ with different residence time $(100,50,40$ and $35 \mathrm{~min}$ ), and the removal rates of TOC were $79.03 \%, 61.64 \%, 52.03 \%$ and $46.67 \%$, respectively. It indicates that the TOC removal rate can be improved by appropriately prolonging the residence time, but the removal ability of pollutants at low flow rate can be significantly reduced [25]. XU et al found that the residence time was shorter with the increase of speed by adjusting peristaltic pump speed (100 rpm 600 rpm), the degradation performance of naproxen was enhanced in circulating flow system. In short, the increase of peristaltic pump speed enhances the mass transfer rate in the filter electrochemical reactor and strengthens the anodic oxidation kinetic efficiency [23].

\section{6. electrolyte system}

The electrolyte system will have a significant impact on the degradation path and reaction kinetics of pollutants, and the corresponding degradation mechanisms may be greatly different under different electrolyte systems. GIRALDO et al carried out the degradation experiments of $\beta$-lactam antibiotics oxacillin, and the degradation paths of oxacillin were explored in four electrolyte systems ( $\mathrm{NaHCO} 3, \mathrm{Na} 2 \mathrm{SO} 4, \mathrm{NaCl}, \mathrm{NaNO} 3$ ). They found that direct oxidation was the most important degradation pathway of oxacillin in $\mathrm{NaHCO} 3$ and $\mathrm{Na} 2 \mathrm{SO} 4$. In NaNO3 system, the alkaline hydrolysis of oxacillin under alkaline conditions $(\mathrm{pH} \geqslant 10)$ was helpful to its removal. Compared with the four systems, they believed that the $\mathrm{NaCl}$ system was the most suitable for the removal of oxacillin. The main degradation path was to indirectly oxidize oxacillin by producing chlorinated oxidation groups (mainly $\mathrm{HOCl}$ ). While in the chloride ion system, oxacillin could be converted into biodegradable substrates in $4 \mathrm{~min}$ with low energy consumption, and the antibacterial effect of oxacillin was lost [26].

\section{Conclusion}

In conclusion, ECMR retains advantages of both membrane filtration and electrochemical advanced oxidation technology and avoids its drawbacks. It has the advantages of membrane fouling, high mass transfer efficiency, low power consumption and high removal rate of antibiotics. However, since the working mechanism of the electrochemical membrane reactors is complicated, a system may also involve multiple principles such as electro-adsorption, electrooxidation, catalytic oxidation, screening, and interception. Therefore, there are still some limitations in the study of the mechanism of antibiotic removal by electrochemical membrane reactor. In addition, how to choose more appropriate electrode materials, how to reduce the development of the membrane electrode costs and how to prolong the service lifetime of electrode are also important research directions. Finally, it should be pointed out that the scale of water treatment of electrochemical membrane reactor is still in the laboratory stage. Therefore, there are still deficiencies in how to amplify the reaction device to meet the application requirements of real scenes.

\section{Acknowledgements}

This paper was funded by China National Critical Project for Science and Technology on Water Pollution Prevention and Control (No. 2018ZX07601002), and the Liaoning Provincial Federation of Social Sciences Funded Project (No. 20201slktyb-075).

\section{References}

1. Y. Jia, S. K. Khanal, H. Zhang, G. H. Chen, H. Lu, Water res. 119, (2017)

2. X. Zhang, W. Guo, H. Ngo, H. Wen, N. Li, W. Wu, J. Environ. Manage. 172, (2016)

3. M. Bojaran, A. Akbari, A. Yunessnia lehi, Polym. Adv. Technol. 30, 7(2019)

4. J. Wang, R. Zhuan. Sci. Total Environ. 701, (2020)

5. P. V. Nidheesh, M. Zhou, M. A. Oturan, Chemosphere 197, (2018)

6. Z. Pan, C. Song, L. Li, H. Wang, Y. Pan, C. Wang, J. Li, T. Wang, X. Feng, Chem. Eng. J. 376, (2019)

7. A. Ronen, S. L. Walker, D. Jassby, Rev. Chem. Eng. 32, 5(2016)

8. H. Hui, H. Wang, Y. Mo, Z. Yin, J. Li, Chem. Eng. J. 376, (2019)

9. M. Chen, C. Wang, Y. Wang, X. Meng, Z. Chen, W. Zhang, G. Tan, Electrochim. Acta 323, (2019)

10. Y. Yin, C. Li, C. Song, P. Tao, M. Sun, Z. Pan, T. Wang, M. Shao, Colloid Surf. A-Physicochem. Eng. Asp. 506, (2016)

11. H. Wang, X. Wei, Y. Zhang, R. Ma, Z. Yin, J. Li, Chin. J. Chem. Eng. 27, 1(2019)

12. Y. Qi, Y. Zhang, Z. Yin, H. Wang, B. He, X. Liang, J. Li, J. Electrochem. Soc. 165, 9(2018)

13. Z. Yin, Y. Zheng, H. Wang, J. Li, Q. Zhu, Y. Wang, N. Ma, G. Hu, B. He, A. Knop-Gericke, R. Schlögl, D. Ma, ACS Nano 11, 12(2017)

14. Z. Lang, M. Zhou, Q. Zhang, X. Yin, Y. Li, Sci. Total Environ. 722, (2020)

15. B. P. Chaplin, Accounts Chem. Res. 52, 3(2019)

16. C. A. Martínez-Huitle, M. Panizza, Curr. Opin. Electrochem. 11, (2018)

17. F. C. Moreira, R. A. Boaventura, E. Brillas, V. J. Vilar, Appl. Catal. B-Environ. 202, (2017)

18. Y. Zhang, S. Zuo, M. Zhou, L. Liang, G. Ren, Chem. Eng. J. 335, (2018)

19. S. Liang, H. Lin, X. Yan, Q. Huang, Chem. Eng. J. 636, (2018)

20. Y. Wang, C. Shen, M. Zhang, B. T. Zhang, Y. Yu, Chem. Eng. J. 296, (2016) 
21. Y. Wang, M. Chen, C. Wang, X. Meng, W. Zhang, Z. Chen, J. Crittenden, Chem. Eng. J. 374, (2019)

22. D. Li, J. Tang, X. Zhou, J. Li, X. Sun, J. Shen, L. Wang, W Han, Chemosphere 149, (2016)

23. L. Xu, X. Ma, J. Niu, J. Chen, C. Zhou, J. Hazard. Mater. 379, (2019)

24. F. Liu, Y. Liu, Q. Yao, Y. Wang, X. Fang, C. Shen, ..., J Xie, Environ. Sci. Technol. 54, 9(2020)

25. G. Ren, M. Zhou, M. Liu, L. Ma, H. Yang, Chem. Eng. J. 298, (2016)

26. A. L. Giraldo, E. D. Erazo-Erazo, O. A. FlórezAcosta, E. A. Serna-Galvis, R. A. Torres-Palma, Chem. Eng. J. 279, (2015) 